\title{
Erratum
}

\section{Quarks and anomalies}

\author{
[Int. J. Mod. Phys. A, Vol. 30, No. 2 (2015) 1530012, \\ DOI: $10.1142 / \mathrm{S} 0217751 \mathrm{X} 15300124]$ \\ R. J. Crewther \\ Department of Physics, University of Adelaide, \\ Adelaide SA 5005, Australia
}

Received 29 January 2015

Published 24 February 2015

The following items are typographical errors in the published article.

Second paragraph on Page 2:

The term $\Sigma^{++}$should be delta $\Delta^{++}$and the sentence should read:

"How can a baryon like $\Delta^{++}$exist as an $S$-wave spin-flavor symmetric state $|u \uparrow u \uparrow u \uparrow\rangle$ if quarks are spin- $\frac{1}{2}$ fermions?"

Last line on Page 4:

The phrase "two years later" should be "four years later" and the sentence should read:

"The choice between canonical and anomalous dimensions would be cleared up by asymptotic freedom ${ }^{32,33}$ four years later."

Second paragraph on Page 9:

The term $\partial_{y}^{\mu}$ should be $\partial_{y}^{\nu}$ and the sentence should read:

"In Eq. (17), a single derivative $\partial_{y}^{\nu}$ produces a product of two delta functions, so it is clear that $\theta$-functions in time cannot be used to construct " $T$ "." 
R. J. Crewther

Equation (40) on Page 12:

The term $J_{5}^{\mu}(0)$ should be deleted and the Equation should read:

$$
G_{\alpha \beta \gamma}(x, y) \underset{x \ll y}{\longrightarrow}\left\{K \epsilon_{\alpha \beta \lambda \mu} x^{\lambda} /\left(3 \pi^{2} u^{2}\right)\right\} R^{\prime}\left(\delta_{\gamma}^{\mu} y^{2}-2 y^{\mu} y_{\gamma}\right) I /(\pi v)^{4} .
$$

References 35, Page 15:

arXiv.1203.1321 should be cited before Mod. Phys. Lett. A:

\section{Reference}

35. R. J. Crewther and L. C. Tunstall, arXiv:1203.1321; Mod. Phys. Lett. A 28, 1360010 (2013); EPJ Web Conf. 73, 03006 (2014); Phys. Rev. D 91, 034016 (2015). 